\title{
Multi-wavelength study of a high velocity event near a sunspot
}

\author{
Chia-Hsien Lin $^{1}$, D. Banerjee ${ }^{2}$, E. O’Shea ${ }^{1}$, and J. G. Doyle ${ }^{1}$ \\ 1 Armagh Observatory, College Hill, Armagh BT61 9DG, N. Ireland, UK \\ e-mail: jgd@star.arm.ac.uk \\ 2 Indian Institute of Astrophysics, Koramangala, Bangalore 560034, India
}

Received 27 September 2005 / Accepted 17 November 2005

\section{ABSTRACT}

Context. Jets with velocities higher than $200 \mathrm{~km} \mathrm{~s}^{-1}$ are often observed in the X-ray spectral range in the solar atmosphere, however, very few such objects are observed in the EUV range.

Aims. We report on an eruptive event observed in the south-west side of a sunspot, within active region, AR0554, in a range of spectral lines formed in the transition region and corona.

Methods. The main data were time-series observations obtained with the Coronal Diagnostic Spectrometer (CDS), in addition to images from the Michelson Doppler Imager (MDI) and Extreme ultraviolet Imaging Telescope (EIT) on board the Solar and Heliospheric Observatory. Additional high resolution images from the Transition Region and Coronal Explorer (TRACE) were also used.

Results. The event brightened a loop connecting the sunspot and a bright-point structure, triggering both a high-speed flow in the loop and a transient, low-speed ambient flow into the sunspot. The energy released is quickly conducted to the chromosphere, as deduced from the TRACE $1600 \AA$ A images and the light curve of He I $522 \AA$ as seen by CDS. The results from our multi-wavelength study further indicates that the effect of the eruptive event may have reached temperatures higher than $2 \times 10^{6} \mathrm{~K}$. Relative Doppler velocities of over $300 \mathrm{~km} \mathrm{~s}^{-1}$ were detected in the transition region line, O v $629 \AA$.

Conclusions. The multi-wavelength analysis indicates that the event is likely due to fast magnetic reconnection in the transition region. The velocities detected are consistent with the results obtained by the evaporation jet model.

Key words. Sun: activity - Sun: UV radiation - Sun: transition region - Sun: atmosphere - Sun: corona

\section{Introduction}

Energetic events with abrupt brightness enhancement and highspeed material motions have long been observed in the solar atmosphere by various instruments. These events are often classified based on observables, such as, line profile variations, the temperature range over which the events are detected, the duration of the event, and the speed of material motion associated with the event.

Although it is well known that these events are the consequences of magnetic reconnection and flux cancellations, it is still not clear where the triggering of the brightening events happen. Understanding the relation between the magnetic activities and the observed brightenings can provide insight to the physical mechanisms of the heating and the energy transport in the solar atmosphere. At the photospheric level, magnetograms have revealed dynamic magnetic activities, many of which have been associated with features observed in the low atmosphere.

Solar surges, often observed in $\mathrm{H} \alpha$, are dark or bright streamers stretching out from sunspot regions (Roy 1973). The material in the solar surge is believed to be dense chromospheric jets moving into the corona (Rust \& Webb 1977). The jets can reach peak velocities of $50-200 \mathrm{~km} \mathrm{~s}^{-1}$ and last for 10-20 min (Canfield et al. 1996).
Another eruptive event in the lower atmosphere are Ellerman bombs (Ellerman 1917). These are small-scale $\left(<5^{\prime \prime}\right)$ and short-lived ( $<20 \mathrm{~min}$ ) phenomena often observed in the upper photosphere and/or lower chromosphere. Their light curves show rapid rising and decaying, and, are symmetric relative to the brightness peak (Qiu et al. 2000). They tend to occur around sunspots. The bombs are associated with different magnetic features, e.g. emerging flux regions, magnetic inversion lines, moving magnetic features (Nindos \& Zirin 1998; Qiu et al. 2000).

In the higher atmosphere, from the upper chromosphere to the corona, eruptive phenomena such as explosive events (or bi-directional jets) and blinkers are commonly detected above the quiet Sun areas with weak and mixed polarity fluxes (Chae et al. 1998; Madjarska \& Doyle 2003), while extreme ultraviolet (EUV) and X-ray jets have been mostly observed above the active regions that contain complicated magnetic loop structures and occurrences of small flares/H $\alpha$ surges (e.g. Schmieder et al. 1988; Chae et al. 1999; Moon et al. 2004).

Although the regions where the explosive events and EUV jets are most commonly seen are different, the velocities of both phenomena are similar. Both are in the range 100-200 $\mathrm{km} \mathrm{s}^{-1}$. For instance, Innes et al. (1997) reported explosive events with 
Doppler velocities $\approx 180 \mathrm{~km} \mathrm{~s}^{-1}$, and Schmieder et al. (1988), by tracing the trajectory of the brightness maximum, suggested a propagation speed of $100-200 \mathrm{~km} \mathrm{~s}^{-1}$ for an EUV jet observed in O v $1371 \AA$.

Brueckner \& Bartoe (1983), using the High Resolution Telescope and Spectrograph (HRTS), detected jets with velocities up to $400 \mathrm{~km} \mathrm{~s}^{-1}$ in the transition-zone lines above a quiet-Sun area. The authors suggested that jets with velocities higher than $400 \mathrm{~km} \mathrm{~s}^{-1}$ may be best observed at coronal temperatures, which were not covered in the spectral range of their instrument.

The statistical study of one hundred X-ray jets by Shimojo et al. (1996) shows that the jets are often associated with small flares and that many are ejected from bright-point like features. The velocities of the jets they studied ranged from $10-1000 \mathrm{~km} \mathrm{~s}^{-1}$ with an average velocity $\sim 200 \mathrm{~km} \mathrm{~s}^{-1}$.

The possible triggering sources of EUV/X-ray jets have been investigated by examining their morphology and characteristics, plus their correlations with $\mathrm{H} \alpha$ surges and other magnetic activities. The investigations have shown different results. Rust \& Webb (1977) found that the intensity enhancement in X-ray loops are better correlated with $\mathrm{H} \alpha$ filament activities than with $\mathrm{H} \alpha$ surges. The authors thus concluded that $\mathrm{H} \alpha$ surges, being cool ejections following pre-existing field lines, are not associated with X-ray jets and the heating of the corona (Rust et al. 1977). They suggested, however, that it is the $\mathrm{H} \alpha$ filament eruptions that are responsible for the brightenings in the coronal loops. Canfield et al. (1996), in contrast, indicate a close association between X-ray jets and $\mathrm{H} \alpha$ surges even though the two events are not co-spatial. The results from Chae et al. (1999) suggest that $\mathrm{H} \alpha$ surges and EUV jets are dynamically connected to each other but are not co-spatial. Using the Yohkoh Soft X-ray Telescope, Shibata et al. (1992a) observed many X-ray jets in the solar corona, which were often associated with flares in X-ray bright points, emerging flux regions or active regions. The results by Wang \& Shi (1993) indicate that the energy released by magnetic activity in the low atmosphere (e.g. H $\alpha$ surges) is insufficient to supply coronal flares and heat the corona. However, the authors pointed out that these lowatmospheric activities play an important role in triggering the eruptive events in the corona.

Except for the results by Brueckner \& Bartoe (1983), the velocities of eruptive events detected in UV/EUV have been below $\approx 200 \mathrm{~km} \mathrm{~s}^{-1}$ while the velocities for X-ray jets can reach up to $1000 \mathrm{~km} \mathrm{~s}^{-1}$. The difference may be due to the difference in the sound speed at different temperatures. Here, using the Normal Incidence Spectrometer (NIS) of CDS, we report the detection of an eruptive event which increased the intensity in several spectral lines, ranging from chromospheric to coronal temperatures, by a factor of three in approximately one minute. Relative Doppler velocities over $300 \mathrm{~km} \mathrm{~s}^{-1}$ were detected in the transition region line, O v $629 \AA$. This is the first report of such high velocities being detected by NIS/CDS. The event brightened a loop connecting the sunspot to a bright-point feature at the west of the sunspot, which matches the characteristic location of X-ray jets by Shimojo et al. (1996). We incorporated images from the Extreme ultraviolet Imaging Telescope (EIT) Fe XII $195 \AA$ channel and the Transition Region And Coronal
Explorer (TRACE) $171 \AA$ and $1600 \AA$ channels, along with Michelson Doppler Imager (MDI) magnetograms, in order to determine the trigger, the location and the extent of this event.

\section{Observations and data calibration}

The data discussed here were obtained from dataset s29526r01, time-series observation from 09:01:24-10:37:14 UT on 12 February 2004 by NIS, one of the components of the Coronal Diagnostic Spectrometer (CDS), on board the Solar and Heliospheric Observatory (SoHO) (Harrison et al. 1995). During the 95-min observation, the pointing of the detector was fixed in space while the Sun rotated under the field of view (FOV). The exposure time was $30 \mathrm{~s}$, and the cadence was approximately $37 \mathrm{~s}$. Our observations used six spectral windows containing the following spectral lines: Si XII $520.8 \AA$, He I $522.2 \AA$, Fe XVI $335.4 \AA$, Fe XVI $360.8 \AA$, Mg IX $368.1 \AA$, Mg X $625.0 \AA$, and O v $629.8 \AA$. The target of the observations was active region AR0554.

To have an overview of the features under investigation, we incorporated images from EIT $195 \AA$, and TRACE $171 \AA$ and $1600 \AA$ passbands. We also used MDI magnetograms to obtain information on the photospheric magnetic fields. The noise and cosmic ray contamination in the TRACE images were reduced by a number of de-spike, de-streak and smoothing routines. The orientations of EIT and MDI images were corrected for the effect of the SoHO instruments been turned $180^{\circ}$ for our observation. In Fig. 1, we show an overview of the active region as seen in TRACE $171 \AA$. Note that there is a dynamic bright point at the south-west side of the sunspot. A close-up view of the structural variation of the bright point over time is further shown in Fig. 2. The loop connecting the bright point (at Solar X -230") to the sunspot was not very prominent at 09:36:54 UT, but became very bright in the image taken at 10:21:50 UT.

NIS/CDS data were calibrated by the most up-to-date standard calibration routines in SolarSoft ${ }^{1}$, with the offset between NIS1 and NIS 2 corrected using the routine nis_rotate. As explained in CDS Software Note No. $53^{2}$, NIS/CDS line profiles were broadened after SoHO's recovery in October 1998. Therefore, each spectral line was fitted by a broadened Gaussian (BGaus) line profile function, and the line intensity was computed accordingly. The errors in the line intensities were computed based on the equations in CDS Software Note No. 49. The relative Doppler velocity, $v_{\mathrm{d}}$, at each pixel along the slit is determined by multiplying the speed of light, $c$, by the relative wavelength difference between the wavelength $\left(\lambda_{i}\right)$ at that point and the average wavelength $\left(\lambda_{\mathrm{av}}\right)$ of the data set, that is, $v_{\mathrm{d}}=c \times\left(\lambda_{i}-\lambda_{\mathrm{av}}\right) / \lambda_{\mathrm{av}}$.

\section{Features detected by different instruments}

In Fig. 3 (top three rows), we show the temporal variations of the line intensities along the detector slit (also called X-T

\footnotetext{
1 http://www.lmsal.com/solarsoft/.

2 http://solar.bnsc.rl.ac.uk/.
} 


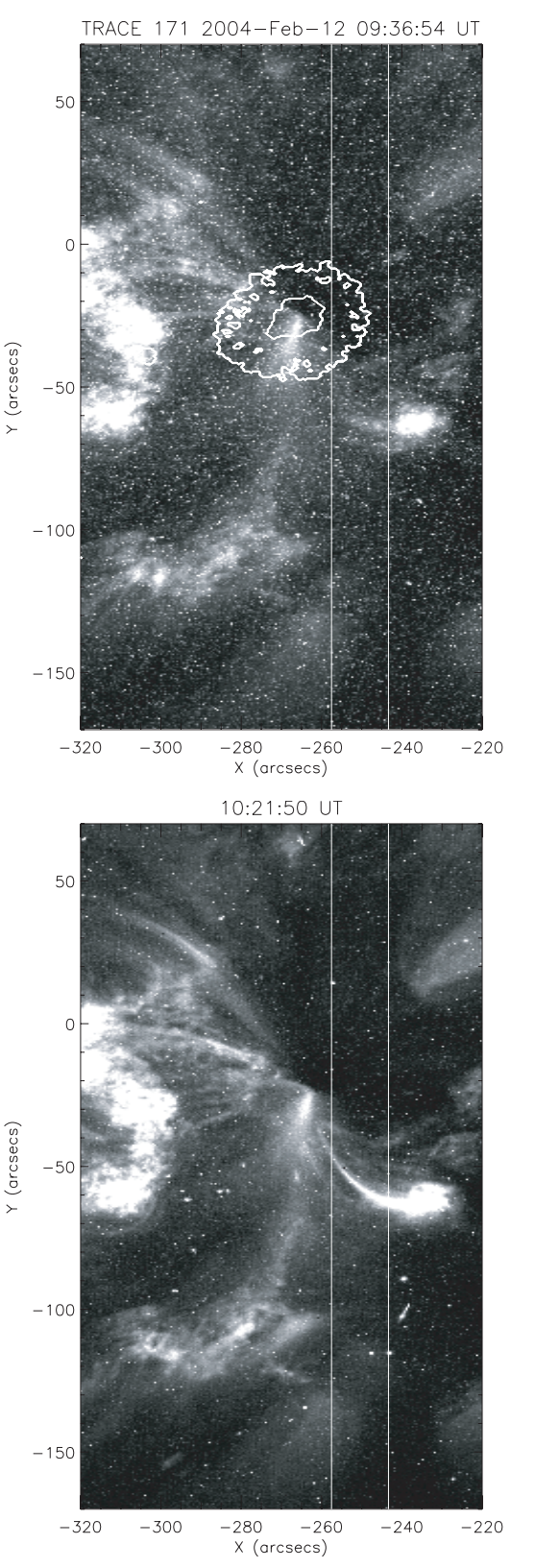

Fig. 1. The TRACE $171 \AA$ channel maps at 09:36:54 UT (upper panel) and 10:21:50 UT (lower panel). North is up and East to the right. The contours in the upper panel mark the boundaries of the umbra and penumbra, and the two parallel lines indicate the NIS/CDS slit locations at the beginning and end of the time-series.

slices). The horizontal is the Solar $Y$ coordinates of the pixels along the slit, and the vertical the time. At approximately 60 min after the start of the observation, we see a sudden brightening in all lines. To quantify the amount of intensity enhancement, we plot in the lower three rows the light curve of a selected pixel, Solar $Y \approx-48^{\prime \prime}$, which is one of the locations showing the large Doppler shifts and intensity enhancement (also see Figs. 4 and 5). The light curves correspond to the location marked by the black dashed line in the X-T slices. The plots show that all six lines increased their intensities by a factor of three in only one time step at $\approx 57.6 \mathrm{~min}$. However, the light curves at different temperatures show distinctive
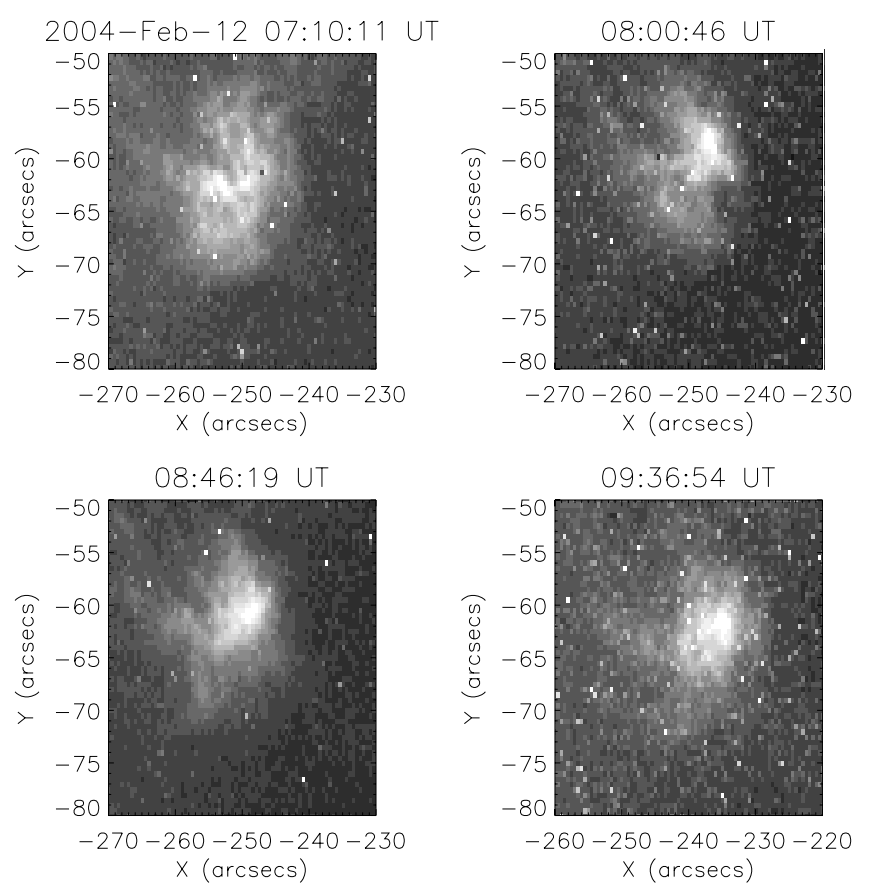

$10: 21: 50$ UT
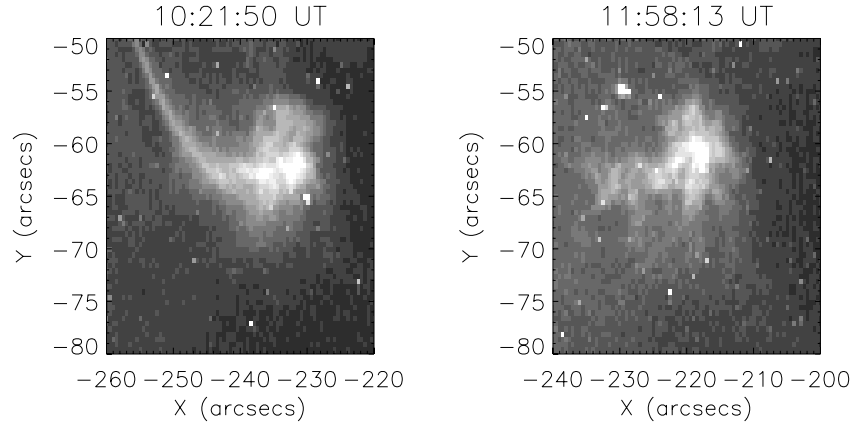

Fig. 2. TRACE $171 \AA$ passband maps showing the structural variation of the bright point over time. The observation time is indicated above each corresponding map.

patterns. The transition-region line, O v $629 \AA$, shows the earliest increase in intensity while the increase in other lines occurs slightly later. The intensity of $\mathrm{O} \mathrm{V}$ increases suddenly and drops very quickly, returning to the average value $\approx 10$ min after the sudden enhancement. The chromospheric line, He I $522 \AA$, demonstrates a sharp intensity increase and a very gradual decaying trend. By the end of the observation, the intensity of He I remains at a high value, as compared to that before the event. $\mathrm{Mg}$ X $625 \AA$ is the only coronal line where the intensity increases sharply. Its intensity reaches the first peak at $\approx 60 \mathrm{~min}$ and a second peak just before $80 \mathrm{~min}$ from the beginning of the observation. The other coronal lines, Si XII $521 \AA$ and Fe XVI 335/361 $\AA$, show a gradual increase and decrease in their intensities. The duration of the whole enhancement-decay process for Si XII and Fe XVI is longer than $20 \mathrm{~min}$.

In Fig. 4 we show the line profiles of O v $629 \AA$ at different locations, which is the only line free from major blending problems. From these profiles, a small deviation from one Broadened-Gaussian fit can be seen in the red wing at five locations along the slit (cf. Fig. 4), one of which is the marked pixel (i.e. $-48^{\prime \prime}$ ) in Fig. 3. Before discussing the possible 

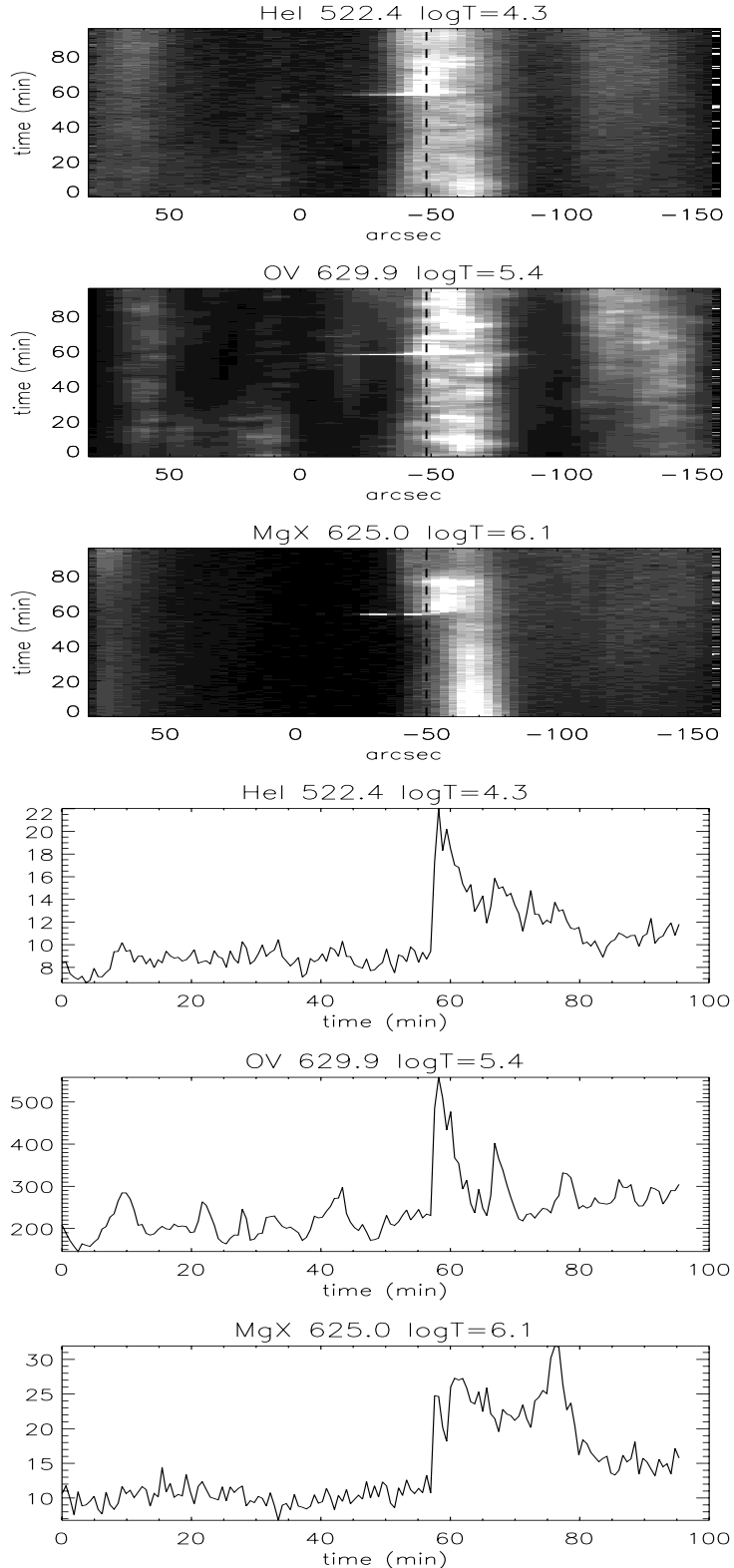
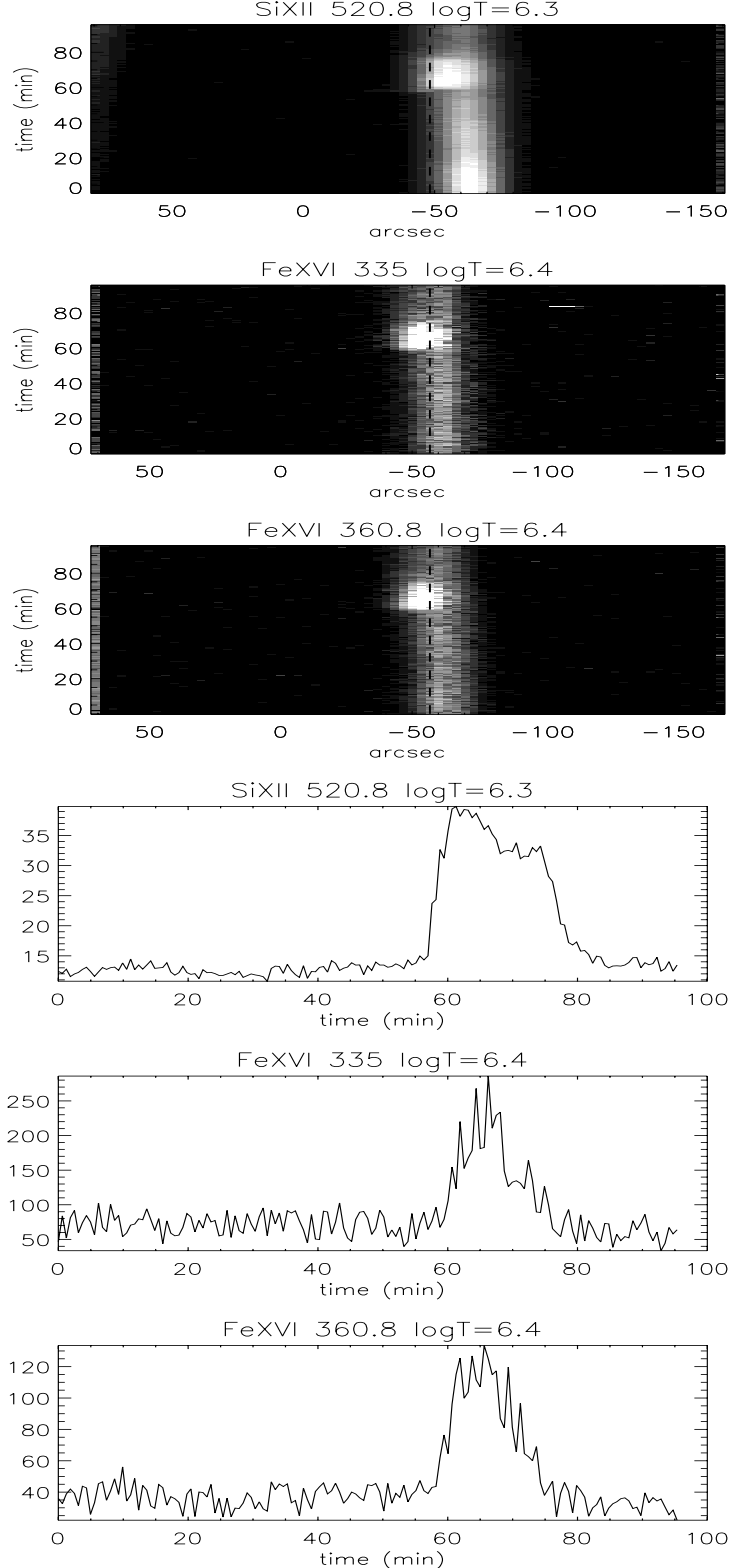

Fig. 3. The top three rows are the $\mathrm{X}$-T slices of the line intensities of the six lines analyzed. The horizontal axis is the Solar $Y$ coordinates along the slit, and the vertical axis is the time. The lower three rows show the line intensity variation along the black dashed lines marked in the X-T slices (Solar $Y \approx-48^{\prime \prime}$ ). The unit of the intensity is photons $/ \mathrm{cm}^{2} / \mathrm{s} / \operatorname{arcsec}^{2}$.

implications of a Doppler-shifted line, we must check for weak line blending. A possible source is Mg VIII $315 \AA$ in second order. Using an active region DEM from CHIANTI, the relative strengths of O V $629 \AA$ and Mg VIII $315 \AA$ is $1.3: 1$ in favour of $\mathrm{O} \mathrm{V}$ for a coronal abundance and 4.3:1 for a photospheric abundance. Based on the second-order efficiency curve (which is a combination of data and theory), the relative efficiency is 25.6 , hence the $\mathrm{O}$ V $629 \AA$ should be a factor of 30 to 100 stronger than Mg VIII $315 \AA$ in second order if the feature does not have an abnormal shaped DEM distribution and/or abundance. Based on the line flux increases shown in Fig. 3 for $\mathrm{O} \mathrm{V}$ and $\mathrm{Mg} \mathrm{X}$, there is no large increase in the coronal line enhancement compared to the transition region line, hence, the red-shifted component is not due to second order line blending but is a result of a Doppler shift component.
The relative Doppler velocity implied from fitting this small deviation is between $230 \mathrm{~km} \mathrm{~s}^{-1}$ and $320 \mathrm{~km} \mathrm{~s}^{-1}$. In Fig. 5, we select pixel $-48^{\prime \prime}$ to illustrate the general pattern of the lineprofile variation with time. The small bump in the red wing is only detectable at $57.6 \mathrm{~min}$. This may be due to the limited wavelength resolution in NIS/CDS, rather than the actual duration of the event. In other words, the bump is only measurable at the time of the sharpest red-shift. The velocity map of the $\mathrm{O} v$ time series shows that in the region of Solar $Y \approx-20^{\prime \prime}$ to $-50^{\prime \prime}$, red-shifts greater than $20 \mathrm{~km} \mathrm{~s}^{-1}$ are detected throughout the whole time series. This indicates that material had been continuously flowing from the bright point into the umbra and that the brightening event caused a sudden surge in the flow. Intriguingly, at the moment of the brightening event, a sudden red-shift greater than $20 \mathrm{~km} \mathrm{~s}^{-1}$ was detected in the region, 

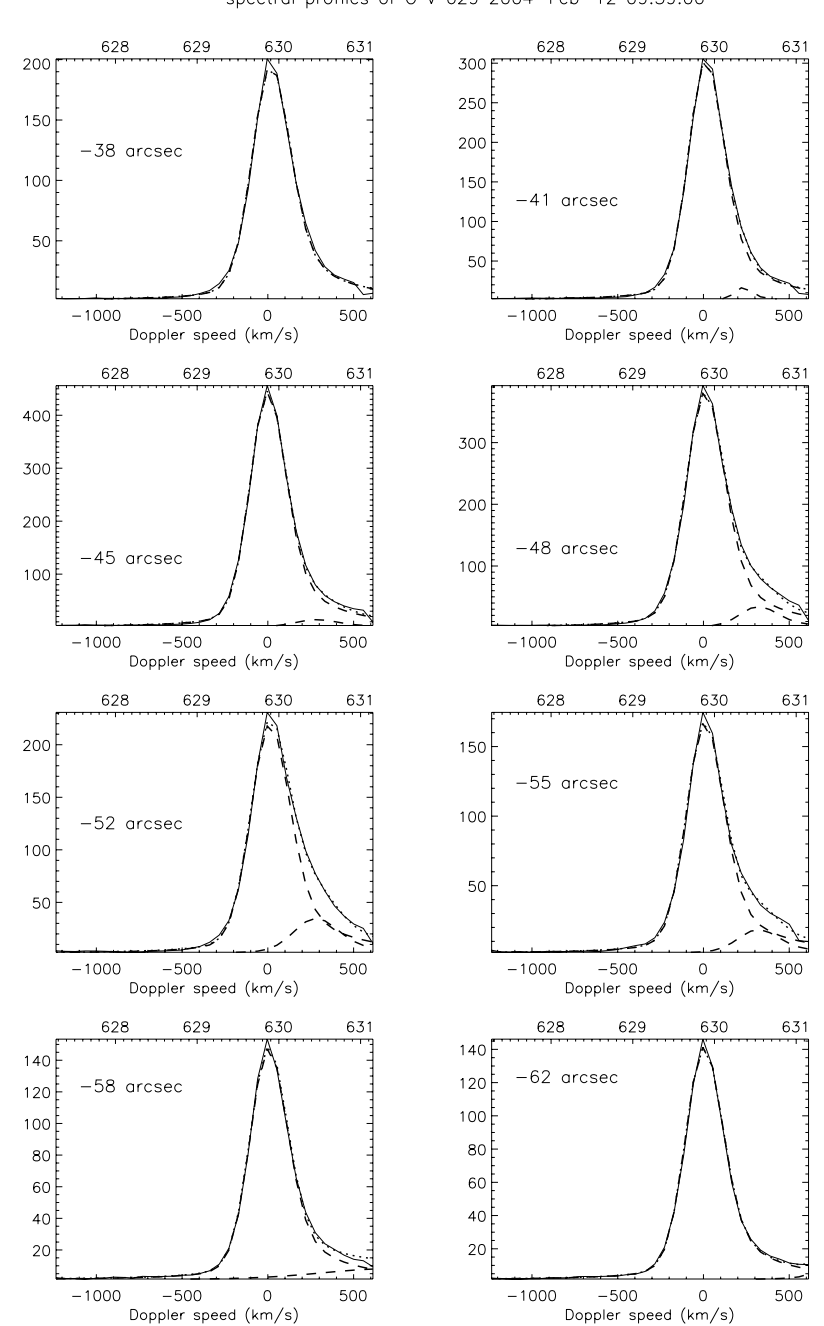

Fig. 4. The line profiles of O v $629 \AA$ at eight locations as indicated in each panel. The line profiles, in units of photons $/ \mathrm{cm}^{2} / \mathrm{s} / \operatorname{arcsec}^{2}$ (CDS Software Note No. 34), are plotted against wavelength, $\AA$, (upper $X$ axis) and the corresponding Doppler speed (lower $X$-axis). The solid lines are the original line profiles, the dotted lines are the fitted profiles, and the dash lines show the profiles of individual broadened Gaussian. The second Broadened-Gaussian fit is apparent from $\approx-41^{\prime \prime}$ to $-55^{\prime \prime}$, which indicates that the large Doppler shifts occur at these locations. This event is recorded at 09:59:00 UT as indicated in the title.

Solar $Y \approx-20^{\prime \prime}$ to $10^{\prime \prime}$. This red-shift only lasted for approximately one minute.

To investigate the origin of the brightening, we examined the MDI magnetograms plus the TRACE $1600 \AA$ and EIT Fe XII $195 \AA$ images, which represent features at the photospheric and coronal levels, respectively. The EIT Fe XII $195 \AA$ images taken around the time of the event are presented in Fig. 6. The figure shows that the bright point and the connecting loop seen in the TRACE $171 \AA$ images (Fig. 1) are also seen at the temperature of EIT Fe XII $195 \AA$. Among all the images in Fig. 6, we can see that the loop is most prominent at 10:24:18 UT, when the bright point is also the brightest. The loop is blurry and fragmented in other images, and the bright point quickly decreases in intensity in the last two
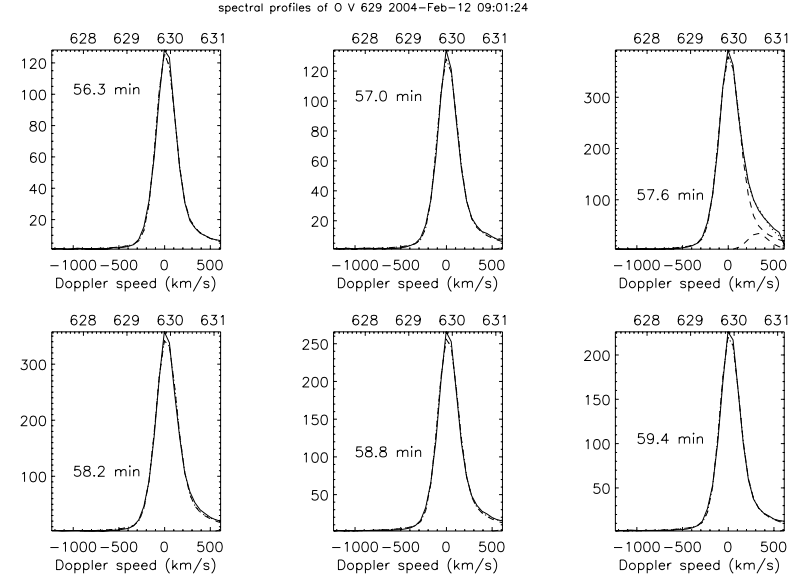

Fig. 5. The temporal variation of line profiles of O v $629 \AA$ at Solar $Y \approx-48^{\prime \prime}$. The time lapse from the beginning of the observation, 09:01:24 UT, is indicated in each panel. The axis and the line profiles are the same as described in Fig. 4. Except for the panel of $57.6 \mathrm{~min}$, the solid line (original profile) is fitted very well by one broadened Gaussian. In the panel of $57.6 \mathrm{~min}$, there is a prominent bump at the red wing of the line, and an additional broadened Gaussian is required to fit the whole line profile.

images, which were taken approximately $11.6 \mathrm{~min}$ and 23.6 min after 10:24:18 UT. Despite the large temporal gaps between the images, the EIT images indicate that, at the temperature of Fe XII $195 \AA$, the brightening occurred between 10:13:55 and 10:24:18 UT. This is consistent with the possible time (before 10:21:50 UT) of the brightening seen in the TRACE $171 \AA$ images (cf. Fig. 1) and also the intensity jump as recorded by NIS/CDS. The six TRACE $1600 \AA$ images in Fig. 7 were selected to illustrate the variations of features at different times. The arrow points to the location of the brightpoint structure, and the square encircles a region that suddenly brightened up in TRACE $1600 \AA$. We can see that the squareenclosed region, which was dark at 09:41:29 UT, shows a sudden and dramatic increase in emission at 10:01:29 UT, when the bright-point region also became the brightest. In spite of the absence of the images between 09:41:29 UT and 10:01:29 UT, we can be certain that the brightening event at the photosphere level happened before or at 10:01:29 UT. The later images indicate that the sudden brightenings in the square-enclosed region and the bright point gradually dispersed afterwards. However, the intensity overall remained significantly higher after than before the brightening. The coordinates of the brightening loop in Fig. 1 and the square-enclosed region in Fig. 7 indicate that the two regions are spatially correlated. Specifically, the sudden brightening is at the lower edge of the penumbra where the left vertical line in Fig. 1 crosses the contour of the penumbra.

The series of MDI magnetograms taken before 10:00:03 UT are shown in Fig. 8. The observation time is indicated above each image. The square in each panel is the region corresponding to the brightening seen in TRACE $171 \AA$ and $1600 \AA$ images. We can see that there are small but continuous magnetic activities in this region. For example, the arrow in the panel of 09:53:03 UT points at a positive region that was invisible one minute earlier. A magnetic canceling feature can 

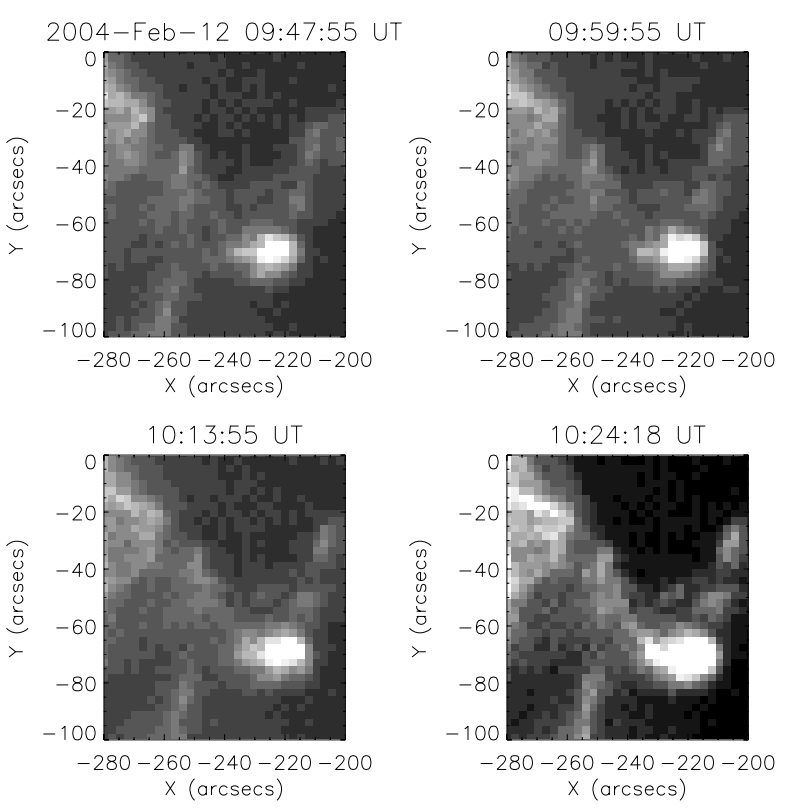

10:35:56 UT
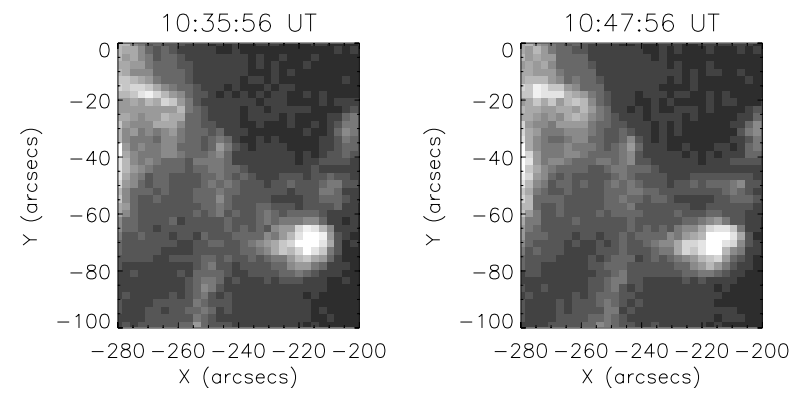

Fig. 6. EIT $195 \AA$ images. The observation time is indicated above each panel. Among all the panels, the bright point became brightest and the loop extending from the left side of the bright point became most prominent at 10:24:18 UT. The last two images, taken approximately $11.6 \mathrm{~min}$ and $23.6 \mathrm{~min}$ later, show that the intensity of the bright point had decreased and the loop had became blurred and fragmented.

be seen between 09:58:03 UT and 10:00:03 UT. The arrow in the panel of 09:58:03 UT points to a negative magnetic region with a positive counterpart at its lower-right side. The negative region was canceled out and became undetectable by 10:00:03 UT. These dynamic magnetic activities may be related to the brightenings seen in the higher temperatures lines. The time delay of the phenomena observed in different temperature layers, as illustrated in Figs. 1, 3, 6-8, seems to suggest that the effect of the photospheric magnetic activities propagates to the higher atmosphere.

\section{Discussion}

Comparisons and associations among solar atmospheric activities in different layers can provide insight to the energy source and propagation in the corona. In the following, we will discuss possible relations among the eruptive event detected by NIS/CDS, the phenomena detected in both the high and low atmosphere, and the magnetic activity at the photospheric level, in the hope of identifying the source of the eruptive event and its extent.
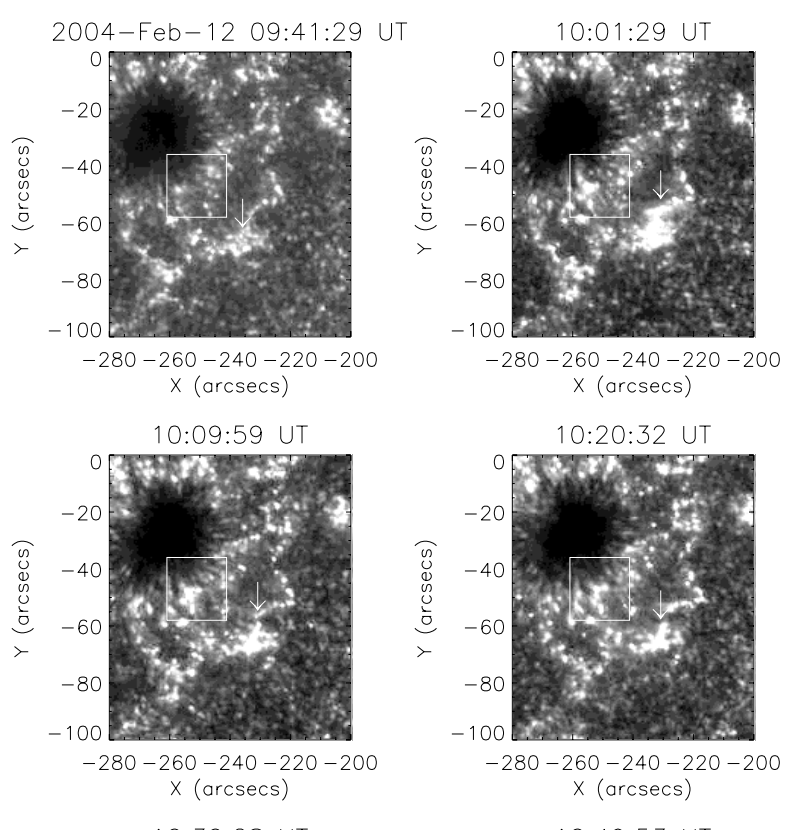

10:30:28 UT
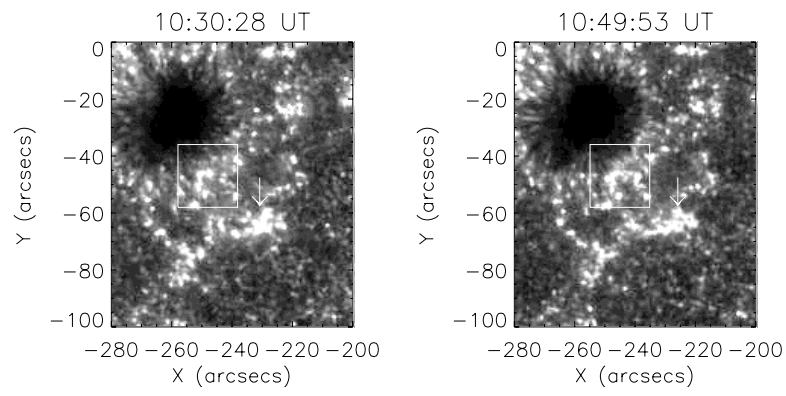

Fig. 7. TRACE $1600 \AA$ channel maps. The regions marked by the square and the arrow were not prominent at 09:41:29 UT, but suddenly became very bright at 10:01:29 UT. The intensity gradually dispersed, and the two bright regions became fragmented after 10:20:32 UT.

The region of interest contains continuous magnetic activities, as evidenced by the MDI magnetograms (Fig. 8). There are small magnetic bipoles constantly emerging and disappearing, which is the manifestation of magnetic reconnection and annihilation. In the TRACE $1600 \AA$ images (Fig. 7), the dark filaments radiating out from the sunspot become well defined after 10:01:29 UT, in which a significant brightening at the boundary of the penumbra is detected (the squared region in Fig. 7). At the left side of the brightening, there is a dark filament protruding southward. This brightening gradually decreases and becomes fragmented by 10:30:28 UT, when the dark filament faded into the background. However, this region remained significantly brighter than before the event happened. The dark filament, stretching out from the sunspot and connecting to the brightening, resembles solar surge phenomena described by Roy (1973). However, the lifetime of the filament, estimated as the time between its first appearance and the moment it faded into the background, is approximately $30 \mathrm{~min}$, which is longer than the typical duration of a surge (10-20 min). As for the brightening, its proximity to the sunspot, correlation with magnetic activities, and the temperature layer where it is detected suggest that it be a potential candidate for an Ellerman bomb (Ellerman 1917; Qiu et al. 2000). However, its size ( $\approx 15^{\prime \prime}$ long 


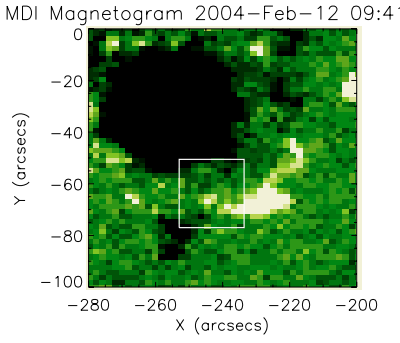

09:53:03

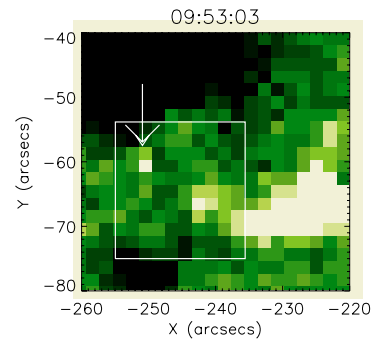

09:57:03

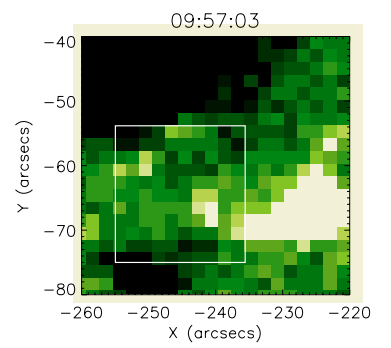

09:42:03

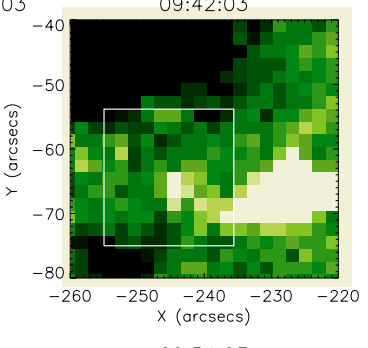

$09 \cdot 54.03$
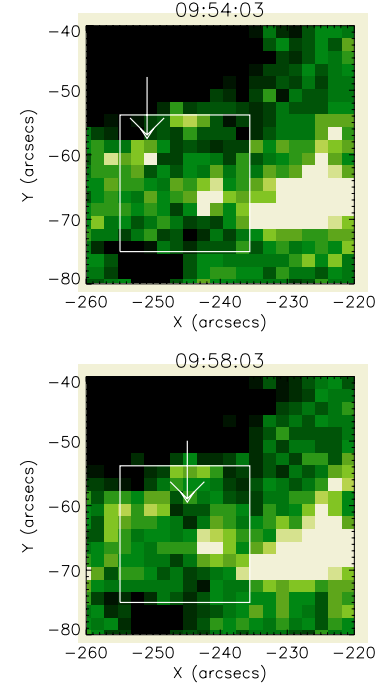

09:51:03

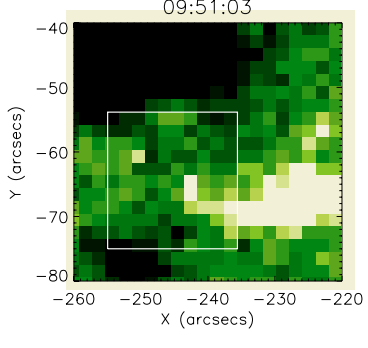

09:55:03

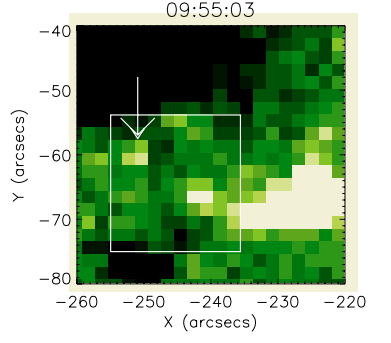

09:59:03

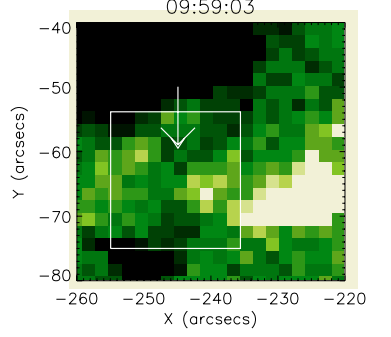

09:52:03

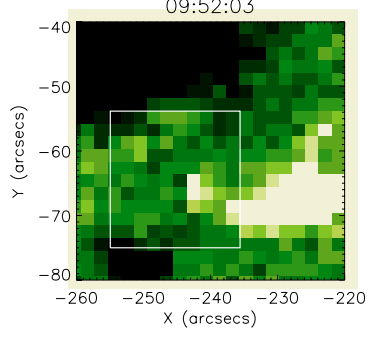

09:56:03

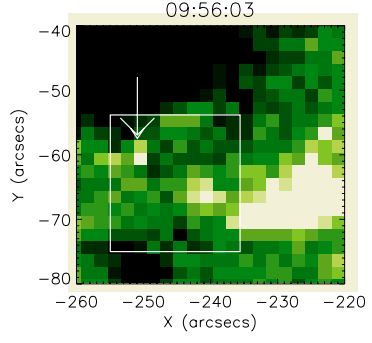

$10: 00: 03$

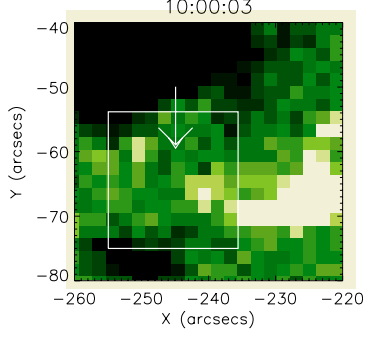

Fig. 8. The MDI magnetograms show the temporal variation of the photospheric magnetic fields. Small but dynamic magnetic fields of opposite polarities can be seen continuously emerging and canceling out in the region enclosed by the white square. The arrows point out two magnetic canceling features. This region overlaps the locations of the line intensity increase detected by NIS/CDS and the brightening seen in TRACE $171 \AA$ and $1600 \AA$ channels.

and $\approx 6^{\prime \prime}$ wide) is too big, and its brightness does not show rapid decay (Qiu et al. 2000); instead, the brightness variation resembles well with the light curve of He I $522.4 \AA$ (cf. Fig. 3), in which the intensity drops slowly and remains higher than the intensity before the event.

The brightening seen in TRACE $1600 \AA$ A could result from either the magnetic activities seen in the MDI magnetograms or the high-velocity, eruptive event detected in NIS/CDS spectral lines, or from both. If the continuous magnetic activities are the cause, there should be either multiple brightenings corresponding to the series of reconnections or a gradual development of brightness resulting from the accumulation of the energy released from the reconnection. Neither of these matches the brightness variation seen in TRACE $1600 \AA$. Therefore, we suspect that the eruptive event detected in the NIS/CDS spectral lines may likely be the source. In addition, comparing Figs. 1 and 7, we can see that the TRACE $1600 \AA$ A brightening coincides with the foot-point of the bright loop seen in TRACE $171 \AA$ A. Therefore, the TRACE $1600 \AA$ brightening may be a manifestation of material flowing down to the footpoint at the chromosphere. The speculation is strengthened by the resemblance between the light curve of He I 522.4 $\AA$ and the brightness variation seen in TRACE $1600 \AA$.

As illustrated in Fig. 3, the temporal variations of the line intensities at different temperatures are distinctively different. The transition-region line, $\mathrm{O} \mathrm{V}$, shows a sharp increase and fastest decrease; the chromospheric line, He I, increases sharply but decreases slowly; the coronal lines, $\mathrm{Mg} \mathrm{X}$ and $\mathrm{Si}$ XII, reaches maximum twice (just after $60 \mathrm{~min}$ and at $\approx 75 \mathrm{~min}$ ) and then gradually decreases; the hotter coronal line, Fe XVI, shows a roughly symmetric profile relative to the intensity apex. In addition, the coronal lines reach their apex at a later time than the transition region line, O V. The Doppler velocities inferred from the line shifts of O V $629 \AA$, ranging from 230 to $320 \mathrm{~km} \mathrm{~s}^{-1}$, are higher than the typical velocity, $\approx 150 \mathrm{~km} \mathrm{~s}^{-1}$, detected in explosive events (e.g. Teriaca et al. 2002), but are in the velocity range $\left(30-400 \mathrm{~km} \mathrm{~s}^{-1}\right)$ of most X-ray jets (Shimojo et al. 1996). However, Brueckner \& Bartoe (1983), analyzing transition-zone spectra from HRTS, reported a type of jet with velocities increasing rapidly with time and reaching up to $400 \mathrm{~km} \mathrm{~s}^{-1}$. The authors suspected that jets of higher velocity might either move out of the field of view of the detector or be heated to coronal temperatures, which were not detectable in the spectral range of their instrument. Their results and the velocities reported in this paper indicate that material at transition-zone temperatures can reach velocities several times greater than the local sound speed. The energy released from the reconnection is quickly dissipated from the site and conducted to the lower atmosphere, in addition to heating the corona, which may explain the fast intensity drop in $\mathrm{O} \mathrm{V}$ and the gradual drop in other lines.

Based on the model proposed by Wang \& Shi (1993), the continuous slow reconnection, as seen in the MDI magnetograms (Fig. 8), can heat the atmosphere and cause the rising 
motion of the magnetic fields (emergence of magnetic flux). The uprising flux presses the overlying large-scale magnetic loops, and creates a current sheet in between. Eventually, an eruptive reconnection happens when a critical state is reached in the current sheet. The velocities we detect are consistent with the results obtained by the evaporation jet model (Shimojo et al. 1996, 2001; Miyagoshi \& Yokoyama 2004). According to the model, both cold and hot plasma are accelerated by the reconnected field lines. The energy released by the ejected hot plasma is conducted to the lower atmosphere. The evaporation flow thus created can reach a maximum velocity $\sim 3 C_{\mathrm{s}} \approx$ $1500\left(T / 10^{7} \mathrm{~K}\right)^{1 / 2} \mathrm{~km} \mathrm{~s}^{-1}$, where $C_{\mathrm{s}}$ and $T$ are the sound speed and temperature of the flow. The velocities obtained from their model are consistent with the observed jet velocity reported here. More recently, the three-dimensional numerical simulations by Archontis et al. (2004) show that the plasma being pushed away by the emerging magnetic flux have velocities $\sim 24 \mathrm{~km} \mathrm{~s}^{-1}$, which is consistent with the red-shift velocities we detected in the surrounding area of the eruptive high-speed event.

Numerical simulations of magnetic reconnection between the emerging flux and overlying horizontal coronal field by Shibata et al. (1992b) show that filament eruptions and cool and hot plasma ejections (i.e. $\mathrm{H} \alpha$ surges and X-ray jets) are all part of the reconnection process. Their model also suggests that $\mathrm{H} \alpha$ surges and X-ray jets can be observed at nearly the same place if the magnetic field and the emerging flux satisfy certain geometries.

\section{Conclusions}

We detected an eruptive event with Doppler red-shift velocities between 230 and $320 \mathrm{~km} \mathrm{~s}^{-1}$ in the south-west side of the sunspot in the active region, AR0554. The high velocities were detected over a spatial extent of $\approx 13^{\prime \prime}$. Such velocities have never been observed by the CDS instrument, and are rarely seen in the EUV spectral range. The event brightened a loop connecting a sunspot and a bright-point structure located at the south-west of the sunspot. The red-shifts and the pointing direction relative to the site of the event indicate that the event resulted in a high-speed flow moving from the bright-point structure into the sunspot. Intriguingly, at the moment of the eruptive event at the south-west side of the sunspot, an abrupt red-shift greater than $20 \mathrm{~km} \mathrm{~s}^{-1}$ was detected at the north-western boundary of the sunspot. This north-western redshift extended from Solar $Y \approx-20^{\prime \prime}$ to $10^{\prime \prime}$, and only lasted for approximately one minute. This sudden in-flow in the west side of the sunspot may be triggered by the eruptive event pushing away the surrounding plasma.

The origin of the event is likely to be a magnetic reconnection in the transition region. The energy released is quickly conducted to the loop foot-point in the chromosphere, as evidenced by TRACE $1600 \AA$ images and the light curve of He I $522 \AA$. The brightening and intensity enhancement detected in the coronal lines indicate that the energy also affected the corona. The results from NIS/CDS indicate that the effect of the eruptive event may have reached temperatures higher than $2 \times 10^{6} \mathrm{~K}$.
Our results are best explained by the emerging flux model proposed by Shibata et al. (1992b), Archontis et al. (2004), and the two-step scenario suggested by Wang \& Shi (1993). Wang $\&$ Shi (1993) suggested that the energy released from the continuous slow magnetic reconnection (first-step reconnection) in the lower atmosphere, can heat the atmosphere and cause magnetic fields rising from the photosphere to the upper atmosphere. When the emerging magnetic flux reaches the coronal level, it presses the overlying coronal magnetic fields. The stress due to the pressure would gradually build up in the interface (a current sheet), and eventually lead to a fast reconnection once a critical state is reached (Shibata et al. 1992b), which is the so-called second-step reconnection by Wang \& Shi (1993). The eruption of the magnetic flux can trigger a down-flow in the neighbourhood by pushing away the pre-existing plasma in the surrounding area as suggested by Archontis et al. (2004).

Acknowledgements. We would like to thank the CDS team for their help in obtaining and reducing the data and Bill Thompson for advice on the line blending. CDS, EIT and MDI are part of SOHO, the Solar and Heliospheric Observatory, which is a project of international cooperation between ESA and NASA. This work was supported in part by a PRTLI research grant for Grid-enabled Computational Physics of Natural Phenomena (Cosmogrid). We wish to thank the Royal Society and the British Council for funding visits between Armagh Observatory and the Indian Institute of Astrophysics.

\section{References}

Archontis, V., Moreno-Insertis, F., Galsgaard, K., Hood, A., \& O'Shea, E. 2004, A\&A, 426, 1047

Brueckner, G. E., \& Bartoe, J.-D. F. 1983, ApJ, 272, 329

Canfield, R. C., Reardon, K. P., Leka, K. D., et al. 1996, ApJ, 464, 1016

Chae, J., Qiu, J., Wang, H., \& Goode, P. R. 1999, ApJ, 513, L75

Chae, J., Wang, H., Lee, C.-Y., Goode, P. R., \& Schuehle, U. 1998, ApJ, 497, L109

Ellerman, F. 1917, ApJ, 46, 298

Harrison, R. A., Sawyer, E. C., Carter, M. K., et al. 1995, Sol. Phys., 162,233

Innes, D. E., Brekke, P., Germerott, D., \& Wilhelm, K. 1997, Sol. Phys., 175, 341

Madjarska, M. S., \& Doyle, J. G. 2003, A\&A, 403, 731

Miyagoshi, T., \& Yokoyama, T. 2004, ApJ, 614, 1042

Moon, Y.-J., Chae, J., Choe, G. S., et al. 2004, J. Korean Astron. Soc., 37,41

Nindos, A., \& Zirin, H. 1998, Sol. Phys., 182, 381

Qiu, J., Ding, M. D., Wang, H., Denker, C., \& Goode, P. R. 2000, ApJ, 544, L157

Roy, J.-R. 1973, Sol. Phys., 28, 95

Rust, D. M., \& Webb, D. F. 1977, Sol. Phys., 54, 403

Rust, D. M., Webb, D. F., \& MacCombie, W. 1977, Sol. Phys., 54, 53

Schmieder, B., Mein, P., Simnett, G. M., \& Tandberg-Hanssen, E. 1988, A\&A, 201, 327

Shibata, K., Ishido, Y., Acton, L. W., et al. 1992a, PASJ, 44, L173

Shibata, K., Nozawa, S., \& Matsumoto, R. 1992b, PASJ, 44, 265

Shimojo, M., Hashimoto, S., Shibata, K., et al. 1996, PASJ, 48, 123

Shimojo, M., Shibata, K., Yokoyama, T., \& Hori, K. 2001, ApJ, 550, 1051

Teriaca, L., Madjarska, M. S., \& Doyle, J. G. 2002, A\&A, 392, 309

Wang, J., \& Shi, Z. 1993, Sol. Phys., 143, 119 